\title{
RESEARCH
}

Open Access

\section{Human umbilical cord-derived mesenchymal stem cells prevent the progression of early diabetic nephropathy through inhibiting inflammation and fibrosis}

E Xiang ${ }^{1,2}$, Bing Han², Quan Zhang², Wei Rao², Zhangfan Wang², Cheng Chang ${ }^{1}$, Yaqi Zhang ${ }^{1}$, Chengshu Tu², Changyong $\mathrm{Li}^{3^{*}}$ and Dongcheng $\mathrm{Wu}^{1,2^{*}}$ (D)

\begin{abstract}
Background: Diabetic nephropathy (DN) is one of the most serious complications of diabetes and the leading cause of end-stage chronic kidney disease. Currently, there are no effective drugs for treating DN. Therefore, novel and effective strategies to ameliorate DN at the early stage should be identified. This study aimed to explore the effectiveness and underlying mechanisms of human umbilical cord mesenchymal stem cells (UC-MSCS) in DN.

Methods: We identified the basic biological properties and examined the multilineage differentiation potential of UC-MSCs. Streptozotocin (STZ)-induced DN rats were infused with $2 \times 10^{6}$ UC-MSCs via the tail vein at week 6. After 2 weeks, we measured blood glucose level, levels of renal function parameters in the blood and urine, and cytokine levels in the kidney and blood, and analyzed renal pathological changes after UC-MSC treatment. We also determined the colonization of UC-MSCs in the kidney with or without STZ injection. Moreover, in vitro experiments were performed to analyze cytokine levels of renal tubular epithelial cell lines (NRK-52E, HK2) and human renal glomerular endothelial cell line (hrGECs).

\footnotetext{
* Correspondence: lichangyong@whu.edu.cn; bcdcwu@hotmail.com ${ }^{3}$ Department of Physiology, Wuhan University School of Basic Medical Sciences, Wuhan, China

'Department of Biochemistry and Molecular Biology, Wuhan University School of Basic Medical Sciences, Wuhan, China

Full list of author information is available at the end of the article
}

(c) The Author(s). 2020 Open Access This article is licensed under a Creative Commons Attribution 4.0 International License, which permits use, sharing, adaptation, distribution and reproduction in any medium or format, as long as you give appropriate credit to the original author(s) and the source, provide a link to the Creative Commons licence, and indicate if changes were made. The images or other third party material in this article are included in the article's Creative Commons licence, unless indicated otherwise in a credit line to the material. If material is not included in the article's Creative Commons licence and your intended use is not permitted by statutory regulation or exceeds the permitted use, you will need to obtain permission directly from the copyright holder. To view a copy of this licence, visit http://creativecommons.org/licenses/by/4.0/ The Creative Commons Public Domain Dedication waiver (http://creativecommons.org/publicdomain/zero/1.0/) applies to the data made available in this article, unless otherwise stated in a credit line to the data. 
(Continued from previous page)

Results: UC-MSCs significantly ameliorated functional parameters, such as 24-h urinary protein, creatinine clearance rate, serum creatinine, urea nitrogen, and renal hypertrophy index. Pathological changes in the kidney were manifested by significant reductions in renal vacuole degeneration, inflammatory cell infiltration, and renal interstitial fibrosis after UC-MSC treatment. We observed that the number of UC-MSCs recruited to the injured kidneys was increased compared with the controls. UC-MSCs apparently reduced the levels of pro-inflammatory cytokines (IL-6, IL-1 $\beta$, and TNF-a) and pro-fibrotic factor (TGF- $\beta$ ) in the kidney and blood of DN rats. In vitro experiments showed that UC-MSC conditioned medium and UC-MSC-derived exosomes decreased the production of these cytokines in high glucoseinjured renal tubular epithelial cells, and renal glomerular endothelial cells. Moreover, UC-MSCs secreted large amounts of growth factors including epidermal growth factor, fibroblast growth factor, hepatocyte growth factor, and vascular endothelial growth factor.

Conclusion: UC-MSCs can effectively improve the renal function, inhibit inflammation and fibrosis, and prevent its progression in a model of diabetes-induced chronic renal injury, indicating that UC-MSCs could be a promising treatment strategy for DN.

Keywords: Diabetic nephropathy, Umbilical cord mesenchymal stem cells, Inflammation, Renal fibrosis

\section{Background}

Diabetic nephropathy (DN) is the most detrimental microvascular complication of diabetes and the leading cause of chronic kidney disease worldwide. Diabetes is a progressive disease. Long-term hyperglycemia causes damage to tissues and organs, resulting in various diabetic complications, such as diabetic retinopathy, diabetic foot, DN, and so on [1]. Among them, DN is a refractory disease with low awareness, high incidence, and high disability. The incidence of DN can reach 30 to $40 \%$ after 20 years of diabetes, of which $5 \sim 10 \%$ of patients will progress to end-stage renal disease, and epidemiological surveys predict that by 2030, DN will become the seventh leading cause of death in the world [2-5]. Risk factors for DN include advanced age, gender, long disease course, obesity, high salt diet, dyslipidemia, nephrotoxic substances, acute kidney injury, and excessive protein intake. Hyperglycemia and hypertension are the most significant risk factors $[6,7]$. Compared with other types of diabetic patients, DN patients have a higher mortality rate, and most of the deaths are due to cardiovascular events [8].

The main manifestation of early $\mathrm{DN}$ is the appearance of microalbuminuria. As the disease progresses, a large amount of proteinuria appears in most patients with DN and eventually develops into chronic renal failure until uremia [9]. The main pathological features of DN include glomerular basement membrane thickening, mesangial expansion, and glomerular sclerosis. Besides, podocyte loss and apoptosis, interstitial inflammation infiltration, renal interstitial fibrosis, and renal tubular atrophy sparse capillaries around the tube are also included in the pathological characteristics of DN [10]. The prevention and treatment of $\mathrm{DN}$ are mainly divided into pre-diabetes prevention (active screening, early detection, and reasonable intervention), early treatment (tight control of blood glucose and blood pressure) to delay the development of DN and comprehensive treatment of advanced DN (including alternative treatments such as dialysis or kidney transplantation) to reduce the risk of cardiovascular events and death [11]. Currently, effective therapeutic strategies to counteract and reverse the progression of $\mathrm{DN}$ are lacking; therefore, it is imperative to develop new strategies for treating DN.

Notably, stem cell therapy has become the most likely new breakthrough in the treatment of DN due to its self-renewal capacity, multilineage differentiation potential, paracrine effects, and immunomodulatory properties. Mesenchymal stem cells (MSCs) are a class of adult stem cells derived from mesoderm that have strong selfrenewal and multi-directional differentiation potential. The main sources are the bone marrow MSCs (BMMSCs), adipose-derived MSCs (AD-MSCs), and umbilical cord MSCs (UC-MSCs). A large number of studies have investigated the role of MSCs in many diseases, including diabetic retinopathy [12], myocardial infarction [13], diabetes [14], and DN [15-19] in mice and rats. However, the underlying mechanisms of these beneficial effects are not completely elucidated.

UC-MSCs are considered to be a better choice of MSCs for clinical applications due to their easy collection, low immunogenicity, and high paracrine potential [20]. In this study, we established streptozotocin (STZ)-induced DN rat models and high glucose-induced HK2 cells (human proximal tubular epithelial cells), NRK-52E cells (rat renal tubular epithelial cells), and hrGECs (human renal glomerular endothelial cells) injury models in vitro and explored the therapeutic effect and underlying mechanisms of UC-MSCs in DN. Our results suggested that early transplantation of UCMSCs holds a promising role in controlling the progress of $\mathrm{DN}$ at an early stage. This study could provide preclinical experimental data for the development of new stem cell drugs for the treatment of $\mathrm{DN}$. 


\section{Materials and methods}

Isolation, culture, and identification of UC-MSCs

The use of the human umbilical cord tissue from a healthy donor who gave birth and signed informed consent in Renmin Hospital was supported by the Institutional Ethics Review Board of Renmin Hospital of Wuhan University (Permit Number: WDRY2019-G001). In a sterile Petri dish, the umbilical cord was cut into small pieces of about $1.0 \mathrm{~mm}$. The tissue fragments were washed with PBS and centrifuged at $1900 \mathrm{r} / \mathrm{min}$ for 6 min. The tissue fragments were resuspended with $25 \mathrm{~mL}$ of serum-free stem cell culture medium (Lonza, MD, Walkersville), inoculated into a T175 culture flask, and placed in an incubator at $37^{\circ} \mathrm{C}$ and $5 \% \mathrm{CO}_{2}$ concentration. The primary cells were harvested when the confluence reached $80 \%$. When the adherent cells were passaged to the fourth generation, after $72 \mathrm{~h}$ of cell culture, the supernatant was collected as UC-MSCconditioned medium (UC-MSC-CM) and centrifuged at $500 \mathrm{~g}$ for $10 \mathrm{~min}$ and passed through a $0.22-\mu \mathrm{m}$ filter (Millipore) before use. The fifth generations of cells were used in this animal experiment. Human UC-MSC induction differentiation experiments were carried out using Oil red $\mathrm{O}$ staining to confirm adipogenesis, using Alizarin red staining to verify osteogenesis, and Alcian blue staining to examine chondrogenesis. Related cell surface markers, such as CD19, CD34, CD45, HLA-DR, CD105, CD90, CD44, and CD73 (Biolegend, USA), were detected by flow cytometry.

\section{Rat DN model and treatment}

Specific pathogen-free male Sprague-Dawley rats (180200 g; 6 weeks old) from Hubei provincial center for disease control and prevention (Wuhan, China) were used in this research. This study was performed in accordance with Guidelines for the Care and Use of Laboratory Animals and the Animal Welfare Act in China and approved by the Committee of Animal Care and Use of Hubei Provincial Center for Food and Drug Safety Evaluation and Animal Experiment (Permit Number: 20200101). Rats were maintained under standard feeding conditions (temperature $20 \pm 2{ }^{\circ} \mathrm{C}$; humidity $45-55 \%$; 12 h light \& dark cycle) and free access to food and water.

All rats were randomly allocated to the control group $(N=5)$ or diabetic group $(N=10)$. We used a wellestablished rat model of DN induced by STZ, as described [21]. Briefly, the diabetic rats were induced by a single intraperitoneal injection of $60 \mathrm{mg} / \mathrm{kg} \mathrm{STZ} \mathrm{(Sigma-}$ Aldrich, MO, St. Louis) (dissolved in $0.1 \mathrm{M}$ citrate buffer, $\mathrm{pH}$ 4.5). The control group received an equal volume of vehicle treatment. The tail blood glucose concentration of rat $\geq 16.7 \mathrm{mmol} / \mathrm{L}$ for 3 consecutive days was confirmed as diabetes. The rat urine was collected using metabolic cages to measure volume and protein concentration on weeks 4 to 6 after STZ treatment. The 24 -h urinary protein $\geq 30 \mathrm{mg} / 24 \mathrm{~h}$ was verified as $\mathrm{DN}$. Then, all DN rats were randomly divided into $\mathrm{DN}+\mathrm{PBS}$ group $(N=5)$ and $\mathrm{DN}+\mathrm{UC}-\mathrm{MSC}$ group $(N=5)$. In $\mathrm{DN}+\mathrm{UC}$-MSC group, $2 \times 10^{6} / 500 \mu \mathrm{L}$ UC-MSCs were injected via tail vein, while the DN + PBS group and control group were received the same volume PBS. Two weeks after UC-MSC treatment, urine samples were collected, and all rats were anesthetized with 2 to $3 \%$ isoflurane and euthanized. Urine samples were used to detect 24 h-urinary protein and urine creatinine (Ucr), and the blood samples were used to measure serum creatinine (Scr) and blood urea nitrogen by assay kits. The creatinine clearance rate $(\mathrm{Ccr})$ was then calculated $(\mathrm{Ccr}=\mathrm{Ucr} / \mathrm{Scr} \times \mathrm{V}, \mathrm{V}: \mathrm{ml} / \mathrm{min}$, urine volume per $\mathrm{mi}$ nute). Kidney tissues were collected for further analysis. Rat DN model and treatment were performed by a single experienced operator in a blinded manner. Completely randomized design and blinding were adopted in animal experiments.

\section{Histological analysis, immunofluorescence, and immunohistochemistry staining}

The kidney was cut longitudinally along the long axis, fixed with $10 \%$ neutral formalin, embedded in paraffin, and cut into 5 - $\mu \mathrm{m}$-thick slices several times in a row. To detect the degree of pathological damage and fibrosis of kidney tissues, hematoxylin and eosin (H\&E), periodic acid-Schiff (PAS), and Masson's trichrome (Masson) staining were used to observe the morphological changes of the kidneys under a light microscope.

For immunofluorescence staining, kidney paraffin slices were dewaxed into water. Then, the antigen was retrieved with EDTA antigen retrieval buffer ( $\mathrm{pH} 8.0$ ). Next, slices were washed with PBS (pH 7.4). After slightly drying, the autofluorescence quencher was added to slices, and the slices were rinsed. BSA was dropwise added to slices and incubated. Then, slices were incubated at $4{ }^{\circ} \mathrm{C}$ overnight with 1:100 diluted rabbit anti-F4/ 80 antibody, 1:100 diluted rabbit anti-Collagen IV antibody and 1:100 diluted rabbit anti-CD3 (Bioss, China), respectively. After washing with $\mathrm{PBS}$, the corresponding secondary antibody (Boster, China) was added to incubate at room temperature for $50 \mathrm{~min}$ in dark. After DAPI staining performing (Solarbio, China), images were detected by a fluorescence microscope (Eclipse Ci-e, Nikon, Japan).

For immunohistochemistry staining, kidney sections were blocked with $3 \% \mathrm{H}_{2} \mathrm{O}_{2}$ in absolute methanol for 30 min and nonspecific sites were blocked with bovine serum albumin (BSA) for $30 \mathrm{~min}$ at room temperature. Then, slices were incubated overnight at $4{ }^{\circ} \mathrm{C}$ with 1:200 diluted mouse anti- $\alpha$-SMA antibody (Boster, China), 1 : 100 diluted rabbit TGF- $\beta$ antibody (Bioss, China), and 1: 
50 diluted MAB1281 antibody (EMD Millipore, USA), respectively. After that, the related goat anti-mouse/ rabbit IgG secondary antibody (HRP-labeled) (Boster, China) was added dropwise and incubated. After washing with PBS and counterstaining with 3, 3'-diamnobenzidine $(\mathrm{DAB})$, then counterstaining with cell nuclei hematoxylin, slices were placed in xylene, concentration gradient alcohol to dehydrate, and images were examined by a light microscope.

\section{Enzyme-linked immunosorbent assay}

The levels of IL-1 $\beta$, IL-6, TGF- $\beta$, and TNF- $\alpha$ in kidney tissues, serum, HK2 cells, NRK-52E cells, and hrGECs were determined. The levels of epidermal growth factor (EGF), fibroblast growth factor (FGF), hepatocyte growth factor (HGF), and vascular endothelial growth factor (VEGF) were detected in UC-MSC-CM and UC-MSCExo. ELISA kits (Mlbio, Shanghai, China) were used following the manufacturer's instructions.

\section{RNA extraction and RT-qPCR assay}

Total RNA was extracted from the kidneys, HK2 cells, NRK-52E cells, and hrGECs using Trizol reagent (Life Technologies, USA). The methods of reverse transcription and RT-qPCR were performed following the manufacturer's instructions (RNA-Solv Reagent). The primer sequences for RT-qPCR are listed in Additional file 1: Table 1 and Table 2. The experimental data were normalized to endogenous housekeeping gene GAPDH with $2^{-\Delta \Delta \mathrm{Ct}}$ method.

\section{Western blot analysis}

About $100 \mathrm{mg}$ of the kidney tissue was lysed in $1 \mathrm{~mL}$ ice-cold RIPA lysis buffer (Beyotime, China) with $1 \mathrm{mM}$ PMSF. The concentration of protein was examined by $\mathrm{BCA}$ assay kits. About $50 \mu \mathrm{g}$ protein samples were loaded in 10\% SDS-PAGE gels and transferred to PVDF membrane (Millipore, USA). After blocking with 5\% skim milk for $1 \mathrm{~h}$, the membrane was respectively incubated overnight at $4{ }^{\circ} \mathrm{C}$ with TGF- $\beta$ (1:1000, Abcam, USA), GAPDH (1:5000, Bioss, China), CD9 (1:1000, Proteintech, USA), Alix (1:1000, Proteintech, USA), and Calnexin (1:1000, Cell Signaling, USA), subsequently washed and incubated with corresponding secondary antibodies for $1 \mathrm{~h}$ at room temperature. Specific protein bands were visualized applying ECL kit.

\section{Isolation and identification of UC-MSC-Exo}

Exosomes isolation and identification were performed as described [22] with modifications. The more detailed description of the exosomes isolation is available in Additional Fig. 2. Exosomes extracted from UC-MSCs (UCMSC-Exo) were observed by transmission electron microscope (TEM) (Tecnai G2 Spirit Bio TWIN, FEI,
USA). Particle size and concentrations were measured by nanoparticle tracking analysis (NTA) (Particle Metrix, Germany). Related markers (CD9, Alix) and negative control (Calnexin) of exosomes were detected by western blot.

\section{Cell culture and treatment}

HK2 cells (human proximal tubular epithelial cells), NRK-52E cells (rat renal tubular epithelial cells), and hrGECs (human renal glomerular endothelial cells) were purchased from the China Center for Type Culture Collection (Wuhan China). HK2 cells were cultured in RPMI 1640 medium containing 10\% fetal bovine serum (FBS; Hyclone, USA), NRK-52E cells were cultured in DMEM-low glucose medium with 10\% FBS, and hrGECs were cultured in DMEM/F12 containing 10\% FBS at $37{ }^{\circ} \mathrm{C}$ and $5 \% \mathrm{CO}_{2}$ concentration in a humidified atmosphere. Cells were seeded into 6-well plates and randomly divided into eight groups: (1) low glucose (LG) group: Dglucose $5.5 \mathrm{mmol} / \mathrm{L}$; (2) high glucose (HG) group: D-glucose $30 \mathrm{mmol} / \mathrm{L}$; (3) HG + 25\% UC-MSC-CM group; (4) $\mathrm{HG}+50 \%$ UC-MSC-CM group; (5) HG + 100\% UCMSC-CM group; (6) $\mathrm{HG}+25 \mu \mathrm{g} / \mathrm{mL} \quad$ UC-MSC-Exo group; (7) $\mathrm{HG}+50 \mu \mathrm{g} / \mathrm{mL} \mathrm{UC}-\mathrm{MSC}$-Exo group; and (8) $\mathrm{HG}+100 \mu \mathrm{g} / \mathrm{mL}$ UC-MSC-Exo group. After $24 \mathrm{~h}$ of coculture, the supernatant and cells were collected for further analysis.

\section{Statistical analysis}

All data are presented as mean \pm S.E.M by Graph Pad prism 5 and were analyzed by unpaired Student's $t$ test with SPSS 19 statistical software (SPSS Inc., Chicago, Illinois). Multiple group comparisons were made using one-way analysis of variance (ANOVA) followed by Bonferroni's post hoc test. $P$ value $<0.05$ was considered significant.

\section{Results}

Induced differentiation ability and biological properties of UC-MSCs

UC-MSCs were derived from umbilical cord tissues, which had various inducing differentiation capabilities and basic cell biological properties. Biological effectiveness experiments confirmed that UC-MSCs could be differentiated into adipogenic, osteogenic, and chondrogenic phenotypes (Fig. 1a). Flow cytometry experiments confirmed that UC-MSCs were positive for CD105 (99.40\%), CD90 (99.63\%), CD44 (99.67\%), CD73 (99.62\%), and negative for CD19 (0.00\%), CD34 (0.00\%), CD45 (0.00\%), and HLA-DR (0.00\%) (Fig. 1b).

\section{In vivo model of DN was induced by STZ}

To explore the therapeutic effect of UC-MSCs on DN, we established a rat model of DN induced by STZ 


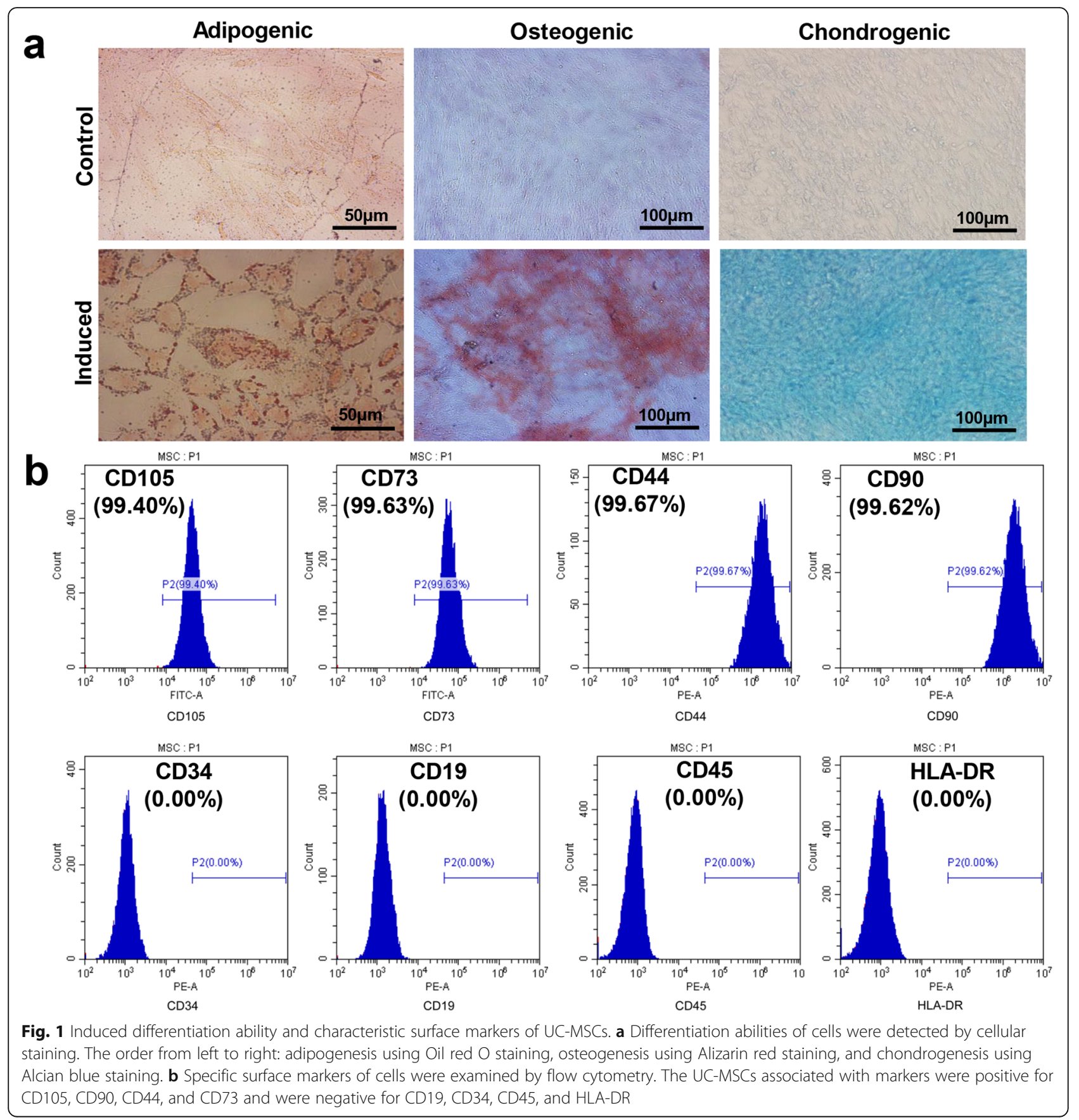

injection. Animals were sacrificed after 2 weeks of treatment, and specimens were collected for further analysis (Fig. 2a). After STZ processing, the bodyweight growth of DN group was significantly lower than that of control group from day 1 to week 6 (Fig. 2b). Besides, the blood glucose levels of DN group were greater than 16.7 $\mathrm{mmol} / \mathrm{L}$ and were significantly higher than that of the control rats (Fig. 2c). The 24-h urinary protein of DN rats was increased and exceeded $30 \mathrm{mg} / 24 \mathrm{~h}$ at week 6 (Fig. 2d), but the Ucr was decreased (Fig. 2e).
Meanwhile, the urinary albumin/creatinine ratio of DN group was significantly increased (Fig. 2f).

\section{UC-MSC treatment improved renal function of DN rats}

In the process of DN modeling and UC-MSC intervention, we observed that DN rats had significantly increased feed and water intake, compared to the control group. Compared with DN rats, UC-MSC-treated DN rats showed a reduction in feed and water intake (Fig. 3b, c). After UC-MSC transplantation, no significant 


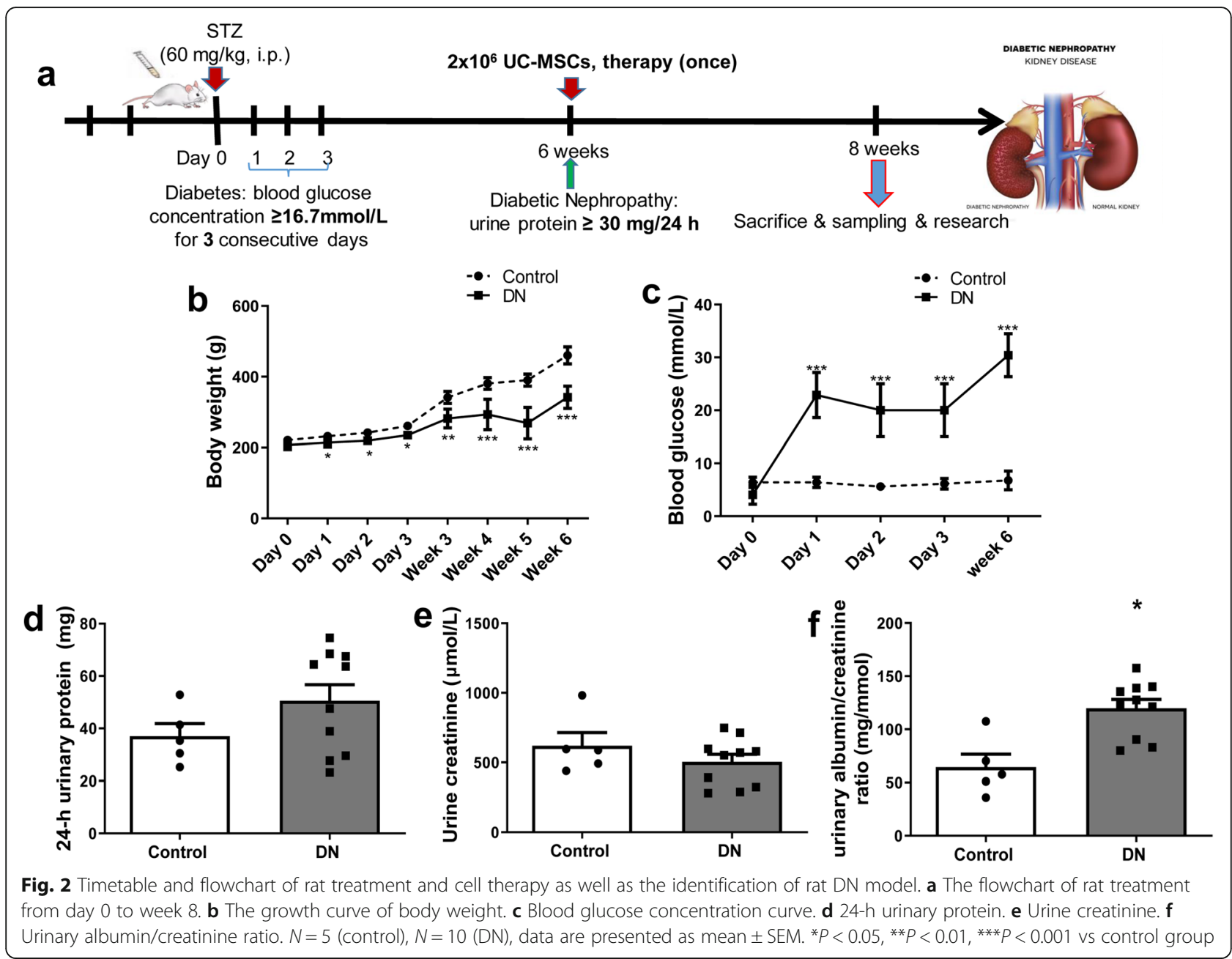

decrease was detected in the blood glucose concentration in DN rats (Fig. 3a). The serum urea nitrogen and Scr of DN group were greatly increased than those of the control group, while UC-MSC treatment significantly suppressed the increase (Fig. 3d, e). Compared with DN group, UC-MSC treatment obviously suppressed the increase in 24-h urinary protein (Fig. 3h) and urinary albumin/creatinine ratio (Fig. 3i), and evidently upregulated Ccr (Fig. 3g). There was a modest but insignificant increase in urine creatinine level on week 8 after UC-MSC infusion (Fig. 3f). These results indicated that UC-MSCs effectively improved renal function in $\mathrm{DN}$ rats.

\section{UC-MSC treatment ameliorated renal pathological changes}

Although we did not detect significant differences in the body weight (Fig. 4a) and kidney weight (Fig. 4b), the kidney weight/kidney weight index was significantly decreased in the DN + UC-MSC group, compared with DN group (Fig. 4c). H\&E staining showed that inflammatory cell infiltration occurred in the renal tubule interstitial tissue and granular degeneration in renal tubular epithelial cells in the DN group. PAS staining showed that the glomerular basement membrane was slightly thickened. Vacuolar degeneration of renal tubular epithelial cells appeared in both $\mathrm{H} \& \mathrm{E}$ and PAS staining of the DN. However, UC-MSC treatment alleviated the above pathological changes (Fig. 4d, e). To confirm whether UC-MSCs have migrated to the kidney tissues, immunohistochemistry staining of MAB1281 and RT-qPCR assay were performed as described [23], to detect the location and distribution of UC-MSCs in the kidneys of rats at 2 weeks after infusion. We observed a small number of MAB1281-positive cells with marked nuclei in brown in the DN + UC-MSC group. Interestingly, MAB1281positive cells mainly localized in tubulointerstitium. No MAB1281-positive cell was found in the control group or DN group (Fig. 5a). The amount of UC-MSCs in the kidney tissues of DN rats was $0.0002 \%$, which was higher than that in the control +UC-MSC group (Fig. 5b). The control group was assayed as a negative control. 


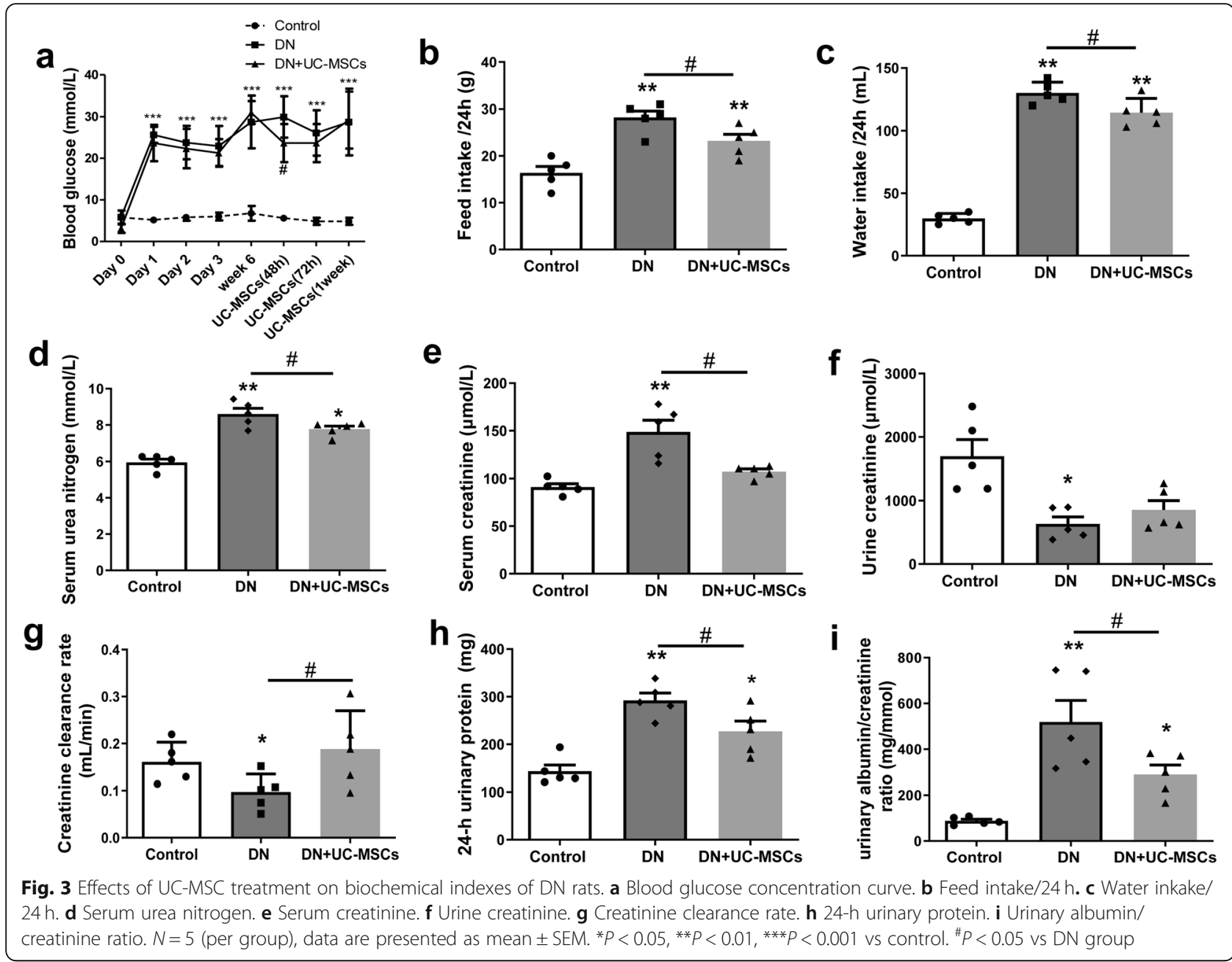

Meanwhile, the content of human-specific DNA fragment HOMO1 in the DN + UC-MSC group was $0.0003 \%$, which was significantly higher than that in the control +UC-MSC group (Fig. 5c).

\section{UC-MSC treatment reduced inflammation in DN rats}

As shown in Fig. $6 \mathrm{a}-\mathrm{c}$, compared to the control group, the mRNA expressions of IL-6, IL-1 $\beta$, and TNF- $\alpha$ (kidney tissues) and the concentrations of IL- 6 , IL- $1 \beta$, and TNF- $\alpha$ (plasma and kidney tissues) were all significantly elevated in DN group, whereas these inflammatory cytokine levels were obviously reduced by UC-MSC treatment compared with DN group. F4/80 and CD3 immunofluorescence staining revealed that the infiltration of inflammatory cells mainly penetrated into the tubulointerstitium in the $\mathrm{DN}$ group, while UC-MSC treatment reduced the above pathological changes (Fig. 6d, e and Additional Fig. 1).

\section{UC-MSC treatment ameliorated renal fibrosis in DN rats}

The Masson's trichrome staining of renal tissues showed that renal glomerular and interstitial fibrosis occurred in the DN group, while the renal fibrosis was improved by UC-MSC treatment in DN + UC-MSC group (Fig. 7a). To further explore the possible role of UC-MSCs in anti-renal fibrosis, we examined the molecules involved in renal fibrosis. The immunofluorescence staining revealed that Collagen IV was majorly expressed in glomerular and tubulointerstitium in the DN group, while the expression of Collagen IV was greatly reduced in DN + UC-MSC group (Fig. 7b, c). Meanwhile, the immunohistochemistry staining showed that the expressions of $\alpha$-SMA and TGF- $\beta$ in the kidneys of DN rats were obviously increased; however, the expression of $\alpha$ SMA and TGF- $\beta$ were decreased after treatment with UC-MSCs (Fig. $7 d$, e). To further confirm that TGF- $\beta$ was involved in renal fibrosis protection, RT-qPCR, ELISA, and Western blot assays were performed. We found that the mRNA (Fig. 7f) and protein (Fig. 7g-i) expression levels of TGF- $\beta$ were markedly enhanced in the DN group, compared to the control group. However, UC-MSC treatment significantly inhibited this enhancement (Fig. $7 \mathrm{f}-\mathrm{i}$ ), further suggesting that the inhibition of 


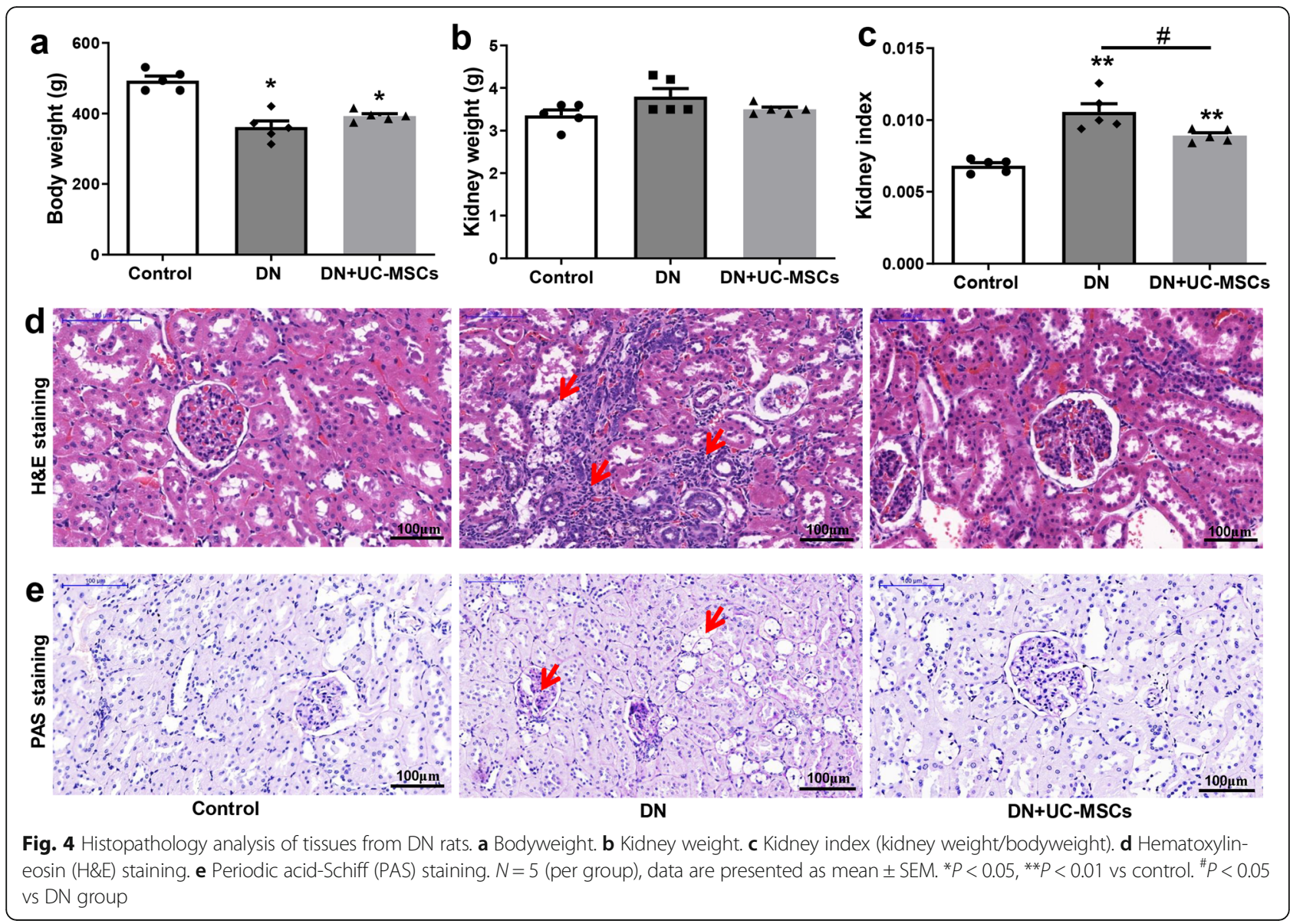

TGF- $\beta$ expression is an underlying mechanism of antirenal fibrosis of UC-MSCs.

\section{UC-MSCs depressed cytokine expression in high glucose-} injured renal tubular epithelial cells and renal glomerular endothelial cells

To further evaluate the anti-inflammatory and antifibrotic effects of UC-MSCs, in vitro experiments were performed in HK2 cells (human proximal tubular epithelial cell line), NRK-52E cells (rat renal tubular epithelial cell line), and hrGECs (human renal glomerular endothelial cell line). UC-MSC-CM was collected, and UCMSC-Exo was isolated and identified (Additional Fig. $2 \mathrm{a}-\mathrm{d})$. Compared with the controls, the mRNA expression and secretion of TGF- $\beta$, IL- 6 , IL- $1 \beta$, and TNF- $\alpha$ were obviously upregulated in high glucose-treated HK2, NRK-52E, and hrGECs (Fig. 8a-h, Additional Figs. 3 and 4). However, UC-MSC-CM or UC-MSC-Exo significantly suppressed high glucose-induced production of TGF- $\beta$, IL-6, IL-1 $\beta$, and TNF- $\alpha$, in a dose-dependent manner (Fig. 8a-h, Additional Fig. 4).

To investigate whether UC-MSCs secreted antiinflammation and anti-fibrosis-related factors, EGF, FGF, HGF, and VEGF levels were measured by ELISA. As shown in Fig. 8i, UC-MSCs secreted large amounts of EGF, FGF, HGF, and VEGF in both UC-MSC-CM and UC-MSC-Exo.

\section{Discussion}

The current clinical routine treatment of DN mainly includes strict blood glucose control and hypertension control to prevent the development of DN. However, despite considerable educational efforts in controlling the disease, a large number of patients still develop not only early and mid-term $\mathrm{DN}$, but also the end-stage chronic kidney disease. Currently, these therapies are still very limited and none of them can effectively cure DN [19]. In this study, we established a STZ-induced $\mathrm{DN}$ rat model and in vitro high glucose-induced HK2 cells, NRK-52E cells, and hrGEC injury models, then performed UC-MSC treatment to explore the efficacy and the underlying mechanisms of UC-MSCs in the treatment of DN. We confirmed that UC-MSCs could migrate to the kidneys and effectively repair renal dysfunction, including reducing the proteinuria, Scr, and urea nitrogen levels and elevating Ccr in DN rats. Importantly, UC-MSCs significantly reduce the inflammatory response and improve renal fibrosis of $\mathrm{DN}$. 


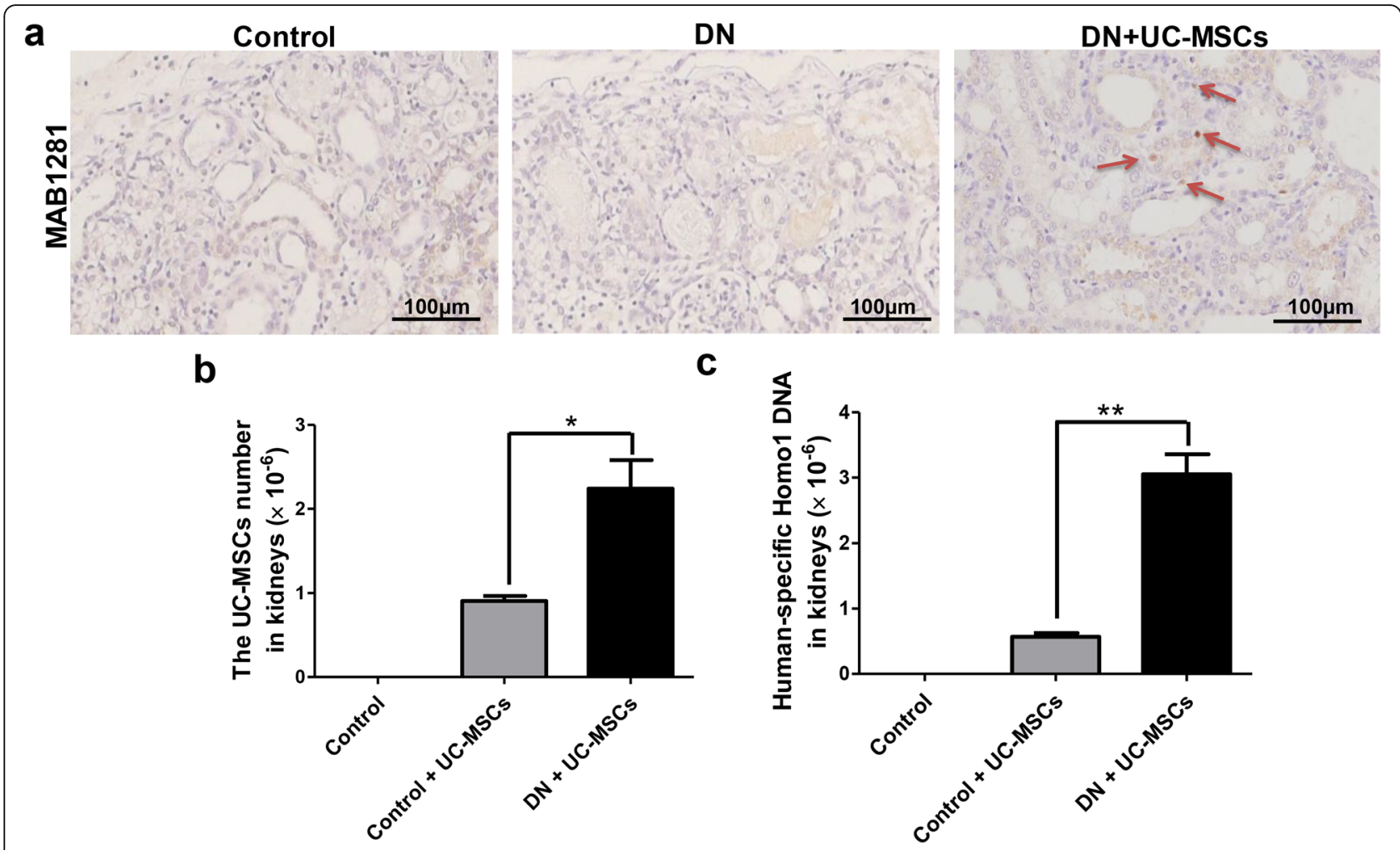

Fig. 5 The location and distribution of UC-MSCs in kidneys of DN rats at 2 weeks after intravenous infusion. a The red arrow indicates MAB1281positive cells in the kidneys of DN rats. $\mathbf{b}$ The number of UC-MSCs detected in the kidneys of DN rats. $\mathbf{c}$ Relative expression of human-specific Homol DNA in kidneys of DN rats. $N=3-4$ (per group), data are presented as mean \pm SEM. ${ }^{*} P<0.05$, ** $P<0.01$ vs control + UC-MSC group

MSCs have many physiological functions, such as selfrenewal, production of clonal cell populations, and multilineage differentiation [20]. In recent years, research on the treatment of DN with MSCs has focused on BMMSCs [24-26]. In clinical applications, there are many problems with the source of BM-MSCs, including the severe pain of BM aspiration and the low number of collected cells, so this has led many researchers to look for other MSCs that are more readily available to replace BM-MSCs. UC-MSCs are a group of young adult MSCs derived from the neonatal umbilical cord tissue. It has the characteristics of the easy collection, greater proliferative capacity, and less antigenicity [20]. UC-MSCs have been used to study a variety of refractory diseases, such as diabetic foot [27], knee osteoarthritis [28], and premature ovarian failure [29]. UC-MSCs are becoming another promising MSCs for treating a variety of human refractory diseases.

Our results showed that, after UC-MSC transplantation, there was no significant change in the blood glucose in DN + UC-MSC group compared with the DN group. This result is consistent with the previous report [30], which may be due to the late treatment of MSCs and the missed opportunity for repairing the acute injury of the pancreas. The islets have been completely destroyed and could not be recovered. We found that 2 weeks after UC-MSC transplantation, compared with the DN group, the 24-h urinary protein concentration, Scr, urea nitrogen, and renal hypertrophy index of the $\mathrm{DN}+\mathrm{UC}-\mathrm{MSC}$ group were significantly reduced, while Ccr was significantly increased, suggesting that UCMSCs had a protective effect on kidney function.

Histological changes in the kidneys are manifested by significant reductions in renal vacuole degeneration, inflammatory cell infiltration, and renal interstitial fibrosis after UC-MSC treatment. Meanwhile, after 2 weeks of intravenous infusion of UC-MSCs, we can detect a small number of cell colonization in rat kidney tissues, and the number of UC-MSCs in DN rats is more than that in normal rats. Previous studies have suggested that when the body ischemia, hypoxia, and injury, MSCs have a "homing" trait that predominately distributes to the injury site [31]. Many researchers believe that the homing ability of MSCs may be the key to their therapeutic effect. Some studies have reported that transplanted MSCs can be tracked in kidney tissues after MSC injection, suggesting that they may be involved in the repair of kidney damage through targeted differentiation or immune regulation [30, 32]. However, it should be noted that the number of "homing" of exogenous MSCs to the 


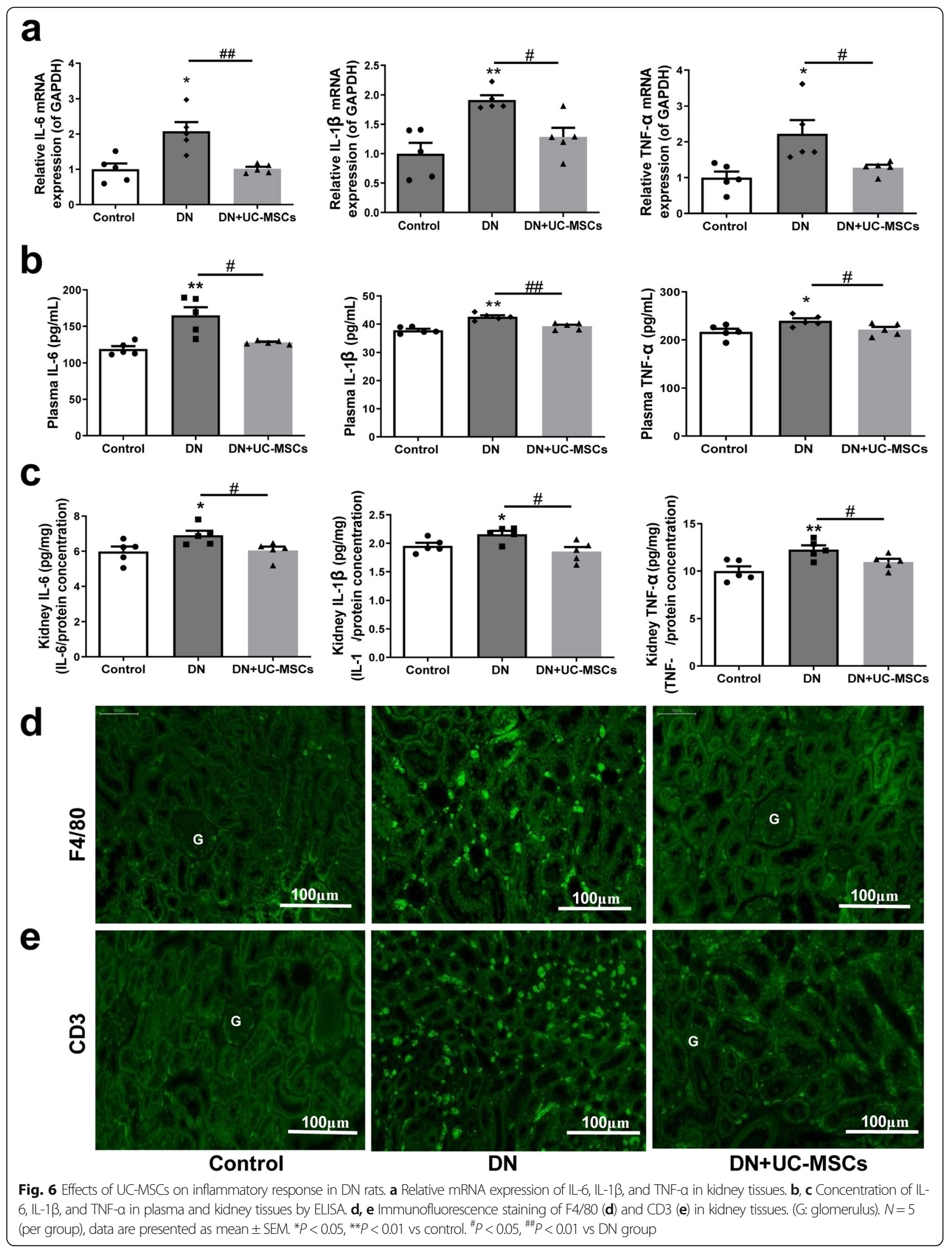




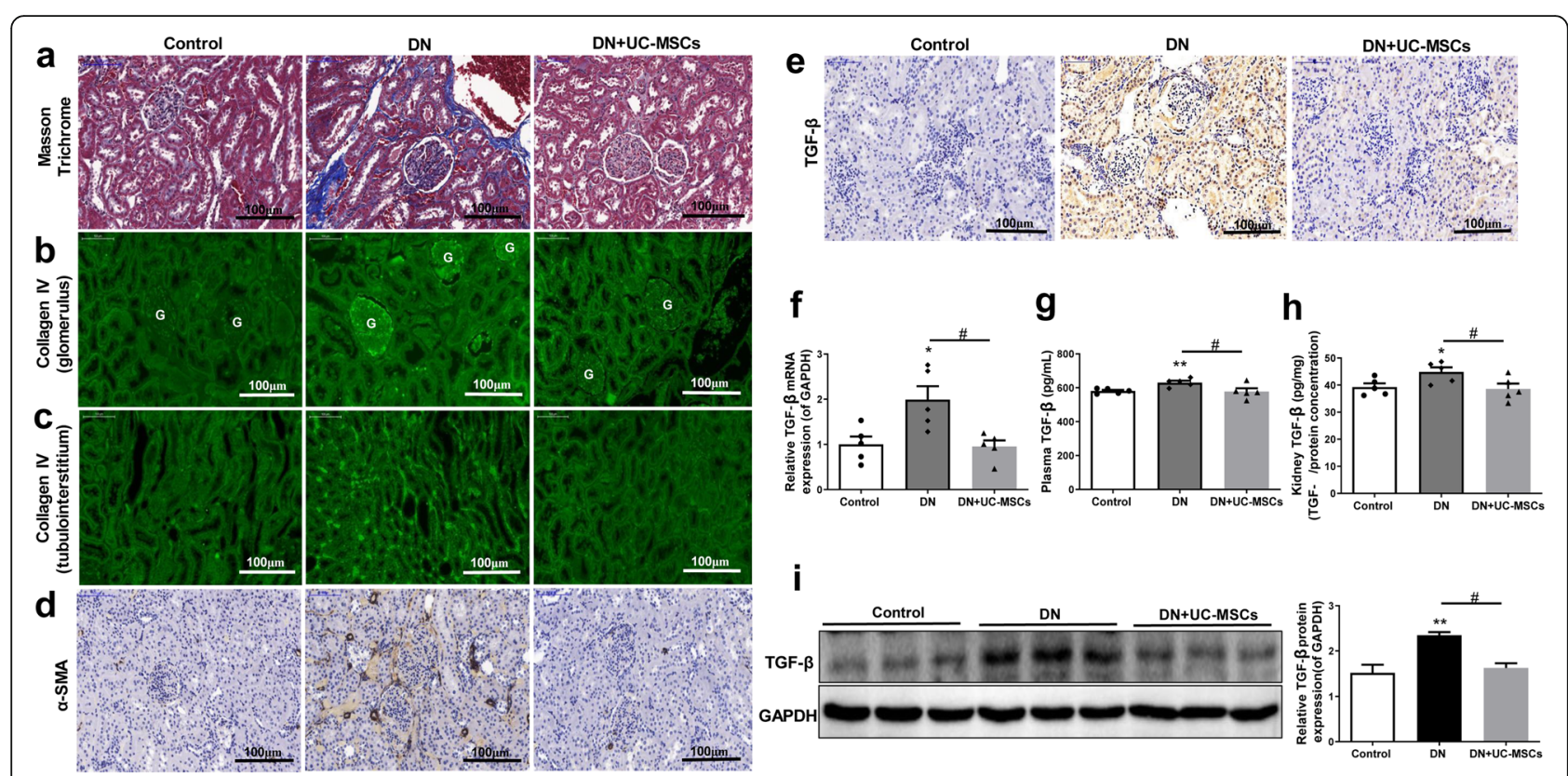

Fig. 7 UC-MSC treatment alleviated glomerular and tubulointerstitial fibrosis in DN rats. a Masson's trichrome staining. b, c Immunofluorescence staining of collagen IV in glomerulus (b) and tubulointerstitium (e). $\mathbf{d}$, e Immunohistochemistry staining of a-SMA (d) and TGF- $\beta$ (e) in kidney tissues. $\mathbf{f}$ The mRNA expression of TGF- $\beta$ in kidney tissues. $\mathbf{g}, \mathbf{h}$ Concentration of TGF- $\beta$ in plasma ( $\mathbf{g}$ ) and kidney (h) tissues. i Western blot analysis of TGF- $\beta$ in kidney tissues. $N=5$ (per group), data are presented as mean \pm SEM. ${ }^{*} P<0.05,{ }^{*} P<0.01$ vs control. ${ }^{\#} P<0.05$ vs DN group

injury site is often limited and does not last long [33]. These results indicated that UC-MSCs can home to kidney tissues and improve the symptoms and pathological changes of DN to a certain extent.

The inflammatory cell infiltration and interstitial fibrosis in $\mathrm{DN}$ are associated with multiple inflammatory cytokines, such as IL-1 $\beta$, IL- 6 , TNF- $\alpha$, and TGF$\beta[21,34,35]$. The expression of IL-1 $\beta$, IL- 6 , and TNF- $\alpha$ was increased in the kidneys of DN rats. UCMSC treatment significantly downregulate the mRNA expression of the above cytokines and reduce the secretion of these cytokines. In addition, UC-MSC treatment inhibited the recruitment of F4/80-labeled macrophages and CD3-labeled lymphocytes in kidney tissues and reduced the inflammatory response in DN rats. Macrophages are the main cellular source of TGF- $\beta$, which interfere with the cell cycle and cause kidney hypertrophy in early DN. TGF- $\beta$ can regulate the gene transcription of myofibroblast phenotype, which is related to the transdifferentiation of renal tubular epithelial cells to fibroblasts and eventually lead to renal interstitial fibrosis [36, 37]. In this study, UC-MSCs obviously reduced the TGF- $\beta$ level and downregulated the renal tubular expression of fibroblast markers, such as $\alpha$-SMA and collagen IV in DN rats. Our results suggest that the improvement effect of UC-MSCs on renal fibrosis in DN rats might be closely associated with the TGF- $\beta$ signaling pathway.
The pathogenesis of DN is extremely complex and a variety of cytokines and growth factors are involved in its formation. Better understanding of the activation of TGF- $\beta$ signaling pathway and its role in DN may provide novel tools for the prevention of renal fibrosis in the future.

To further elucidate the effect of UC-MSCs on DN, we performed in vitro experiments with high glucose-injured HK2 cells, NRK-52E cells, and hrGECs. As expected, we observed that high glucose stimulated the release of proinflammatory cytokines (IL-6, IL-1 $\beta$, TNF- $\alpha$ ) and profibrotic factor (TGF- $\beta$ ) in HK2, NRK-52E, and hrGECs; however, UC-MSC-CM and UC-MSC-Exo significantly decreased high glucose-induced production of these cytokines in a dose-dependent manner. These results suggested that UC-MSCs suppressed inflammation and fibrosis via a paracrine mechanism. To further confirm this issue, we detected certain growth factors that are helpful for anti-inflammation and anti-fibrosis, including EGF, FGF, HGF, and VEGF. Our results indicated that UC-MSCs secreted large amounts of EGF, FGF, HGF, and VEGF in the UC-MSC-CM and UC-MSC-exo. Indeed, previous studies have reported that these growth factors exert anti-fibrotic and anti-inflammatory effects [38-40]. According to our observation in this study and other groups, we speculate that UC-MSC-derived EGF, FGE, HGF, and VEGF might participate in the antiinflammatory and anti-fibrotic effect of UC-MSCs on DN. 


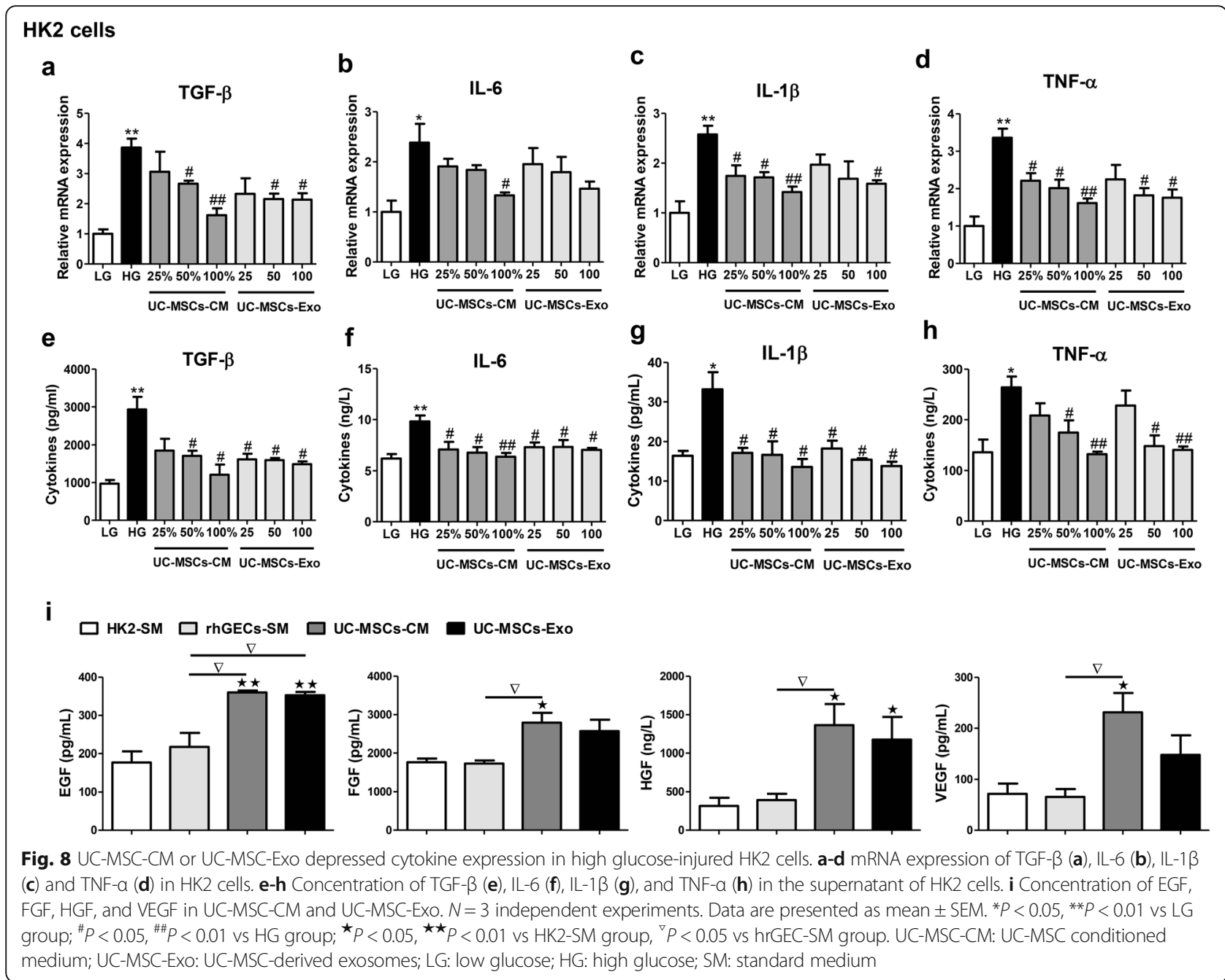

Further studies are required to shed light on the potential involvement and mechanism of these grow factor endocrine and paracrine profiles in DN. Our results indicate that reducing these inflammatory factors and fibrosis factor in DN kidneys can help improve renal function and greatly help understand the role of UC-MSCs in repairing renal function in $\mathrm{DN}$.

\section{Conclusion}

Our study demonstrated that UC-MSCs can effectively improve the renal function, inhibit inflammation and fibrosis, and prevent the progression of early DN. These findings provide a basis for the clinical use of UCMSCs as a new therapeutic approach for DN. Further studies are needed to clarify the relationship between pharmacokinetics and efficacy/toxicity of UC-MSCs in $\mathrm{DN}$ and confirm the precise mechanisms of the antiinflammatory and anti-fibrotic effects of UC-MSCs in improving the occurrence and development of $\mathrm{DN}$.

\section{Supplementary information}

Supplementary information accompanies this paper at https://doi.org/10. 1186/s13287-020-01852-y.

Additional file 1: Table S1. Primers sequences and RT-qPCR conditions for rat genes. Table S2. Primers sequences and RT-qPCR conditions for human genes.

Additional file 2: Figure S1. Immunofluorescence staining of F4/80 and CD3 in the glomerulus of DN kidney tissues. G: glomerulus. Figure S2. Isolation and identification of exosomes extracted from UCMSCS (UC-MSCs-Exo). Figure S3. UC-MSCs-CM depressed cytokine expression in high glucose-injured NRK-52E cells. Figure S4. UC-MSCs-CM or UC-MSCs-Exo depressed cytokine expression in high glucose-injured hrGECs.

\section{Abbreviations}

DN: Diabetic nephropathy; MSCs: Mesenchymal stem cells; UC-MSCs: Human umbilical cord MSCs; BM-MSCs: Bone marrow MSCs; AD-MSCs: Adiposederived MSCS: STZ: Streptozotocin; UC-MSC-CM: UC-MSC-conditioned medium; UC-MSC-Exo: UC-MSC exosomes; HG: High glucose; LG: Low glucose; IL-6: Interleukin 6; IL-1 $\beta$ : Interleukin 1 $\beta$; TNF-a: Tumor necrosis factora; TGF- $\beta$ : Transforming growth factor $\beta$; a-SMA: a-Smooth muscle actin; MAB1281: Mouse anti-human nuclei monoclonal; HOMO1: Human-specific DNA fragment; NRK-52E: Renal tubular epithelial cells; HK2 cells: Human 
proximal tubular epithelial cells; hrGECs: Human renal glomerular endothelial cells; EGF: Epidermal growth factor; FGF: Fibroblast growth factor: HGF: Hepatocyte growth factor; VEGF: Vascular endothelial growth factor; SM: Standard medium

\section{Acknowledgements}

We thank Cuihong Xiao for providing cell experiment help and Jing He, Lian Chen, and Mengting Liu for their help in animal experiments.

\section{Authors' contributions \\ Participated in research design: E Xiang, Changyong Li, Dongcheng Wu, and Bing Han. \\ Conducted experiments: E Xiang, Bing Han, Quan zhang, Wei Rao, Zhangfan Wang, Cheng Chang, Yagi Zhang, and Chengshu Tu. Performed data analysis: E Xiang, Changyong Li, and Dongcheng Wu. Wrote or contribution to the writing of the manuscript: E Xiang, Changyong Li, and Dongcheng $\mathrm{Wu}$. The authors read and approved the final manuscript.}

\section{Funding}

This work was supported by grant from the Wuhan Municipal Science and Technology Bureau (2019030703011513)

\section{Availability of data and materials}

All data generated and/or analyzed during this study are included in this published article.

\section{Ethics approval and consent to participate}

The care and use of animals were approved by the Committee of Animal Care and Use of Hubei Provincial Center for Food and Drug Safety Evaluation and Animal Experiment (Permit No: 20200101). Human umbilical cordderived MSCs were obtained following informed, written patient consent, and the approval was obtained by the Institutional Ethics Review Board of Renmin Hospital of Wuhan University (permit No: WDRY2019-G001).

\section{Consent for publication}

Not applicable.

\section{Competing interests}

The authors declare that they have no competing interests.

\section{Author details}

'Department of Biochemistry and Molecular Biology, Wuhan University School of Basic Medical Sciences, Wuhan, China. ${ }^{2}$ Wuhan Hamilton Biotechnology Co., Ltd, Wuhan, China. ${ }^{3}$ Department of Physiology, Wuhan University School of Basic Medical Sciences, Wuhan, China.

Received: 21 April 2020 Revised: 1 July 2020

\section{Accepted: 24 July 2020 Published online: 03 August 2020}

\section{References}

1. Forbes JM, Cooper ME. Mechanisms of diabetic complications. Physiol Rev. 2013:93(1):137-88.

2. Mathers $C D$, Loncar $D$. Projections of global mortality and burden of disease from 2002 to 2030. PLoS Med. 2006;3(11):e442

3. Saran $R$, et al. US renal data system 2019 annual data report: epidemiology of kidney disease in the United States. Am J Kidney Dis. 2020;75(1 Suppl 1):A6-7.

4. Martinez-Castelao A, et al. The concept and the epidemiology of diabetic nephropathy have changed in recent years. J Clin Med. 2015:4(6):1207-16.

5. Hadjadj $\mathrm{S}$, et al. Death, end-stage renal disease and renal function decline in patients with diabetic nephropathy in French cohorts of type 1 and type 2 diabetes. Diabetologia. 2016;59(1):208-16.

6. Alicic RZ, Rooney MT, Tuttle KR. Diabetic kidney disease: challenges, progress, and possibilities. Clin J Am Soc Nephrol. 2017;12(12):2032-45.

7. Sacks FM, et al. Association between plasma triglycerides and high-density lipoprotein cholesterol and microvascular kidney disease and retinopathy in type 2 diabetes mellitus: a global case-control study in 13 countries Circulation. 2014:129(9):999-1008.

8. Fox CS, et al. Associations of kidney disease measures with mortality and end-stage renal disease in individuals with and without diabetes: a metaanalysis. Lancet. 2012;380(9854):1662-73.
9. Doshi SM, Friedman AN. Diagnosis and management of type 2 diabetic kidney disease. Clin J Am Soc Nephrol. 2017:12(8):1366-73.

10. Espinel E, et al. Renal biopsy in type 2 diabetic patients. J Clin Med. 2015; 4(5):998-1009.

11. Andresdottir $\mathrm{G}$, et al. Improved survival and renal prognosis of patients with type 2 diabetes and nephropathy with improved control of risk factors. Diabetes Care. 2014;37(6):1660-7.

12. Fiori A, et al. Mesenchymal stromal/stem cells as potential therapy in diabetic retinopathy. Immunobiology. 2018;223(12):729-43.

13. Zou L, et al. Bone marrow mesenchymal stem cell-derived exosomes protect against myocardial infarction by promoting autophagy. Exp Ther Med. 2019;18(4):2574-82.

14. Li G, et al. Bone marrow-derived mesenchymal stem cells restored high-fatfed induced hyperinsulinemia in rats at early stage of type 2 diabetes mellitus. Cell Transplant. 2020:29:963689720904628.

15. Lee SE, et al. Mesenchymal stem cells prevent the progression of diabetic nephropathy by improving mitochondrial function in tubular epithelial cells. Exp Mol Med. 2019;51(7):77.

16. Asanuma $\mathrm{H}$, et al. Arterially delivered mesenchymal stem cells prevent obstruction-induced renal fibrosis. J Surg Res. 2011;168(1):e51-9.

17. Li H, et al. Paracrine effect of mesenchymal stem cell as a novel therapeutic strategy for diabetic nephropathy. Life Sci. 2018;215:113-8.

18. Khalilpourfarshbafi $M$, et al. Mesenchymal stem cell-based therapies against podocyte damage in diabetic nephropathy. Tissue Eng Regen Med. 2017; 14(3):201-10.

19. Zhang $L$, et al. Repeated systemic administration of human adipose-derived stem cells attenuates overt diabetic nephropathy in rats. Stem Cells Dev. 2013;22(23):3074-86

20. Wang M, Yuan Q, Xie L. Mesenchymal stem cell-based immunomodulation: properties and clinical application. Stem Cells Int. 2018;2018:3057624.

21. Bai Y, et al. Mesenchymal stem cells reverse diabetic nephropathy disease via lipoxin A4 by targeting transforming growth factor beta (TGF-beta)/ smad pathway and pro-inflammatory cytokines. Med Sci Monit. 2019;25: 3069-76.

22. Thery C, et al. Minimal information for studies of extracellular vesicles 2018 (MISEV2018): a position statement of the International Society for Extracellular Vesicles and update of the MISEV2014 guidelines. J Extracell Vesicles. 2018:7(1):1535750

23. McBride C, Gaupp D, Phinney DG. Quantifying levels of transplanted murine and human mesenchymal stem cells in vivo by real-time PCR. Cytotherapy. 2003;5(1):7-18.

24. Konari $\mathrm{N}$, et al. Mitochondria transfer from mesenchymal stem cells structurally and functionally repairs renal proximal tubular epithelial cells in diabetic nephropathy in vivo. Sci Rep. 2019:9(1):5184.

25. Pan $\mathrm{XH}$, et al. Bone-marrow mesenchymal stem cell transplantation to treat diabetic nephropathy in tree shrews. Cell Biochem Funct. 2014;32(5):453-63.

26. Han JW, et al. Bone marrow-derived mesenchymal stem cells improve diabetic neuropathy by direct modulation of both angiogenesis and myelination in peripheral nerves. Cell Transplant. 2016;25(2):313-26.

27. Cil N, et al. Effects of umbilical cord blood stem cells on healing factors for diabetic foot injuries. Biotech Histochem. 2017:92(1):15-28.

28. Dilogo, I.H., et al., Umbilical cord-derived mesenchymal stem cells for treating osteoarthritis of the knee: a single-arm, open-label study. Eur J Orthop Surg Traumatol, 2020

29. Lu X, et al. The effects of human umbilical cord-derived mesenchymal stem cell transplantation on endometrial receptivity are associated with Th1/Th2 balance change and uNK cell expression of uterine in autoimmune premature ovarian failure mice. Stem Cell Res Ther. 2019;10(1):214.

30. Ezquer $F$, et al. Endovenous administration of bone-marrow-derived multipotent mesenchymal stromal cells prevents renal failure in diabetic mice. Biol Blood Marrow Transplant. 2009;15(11):1354-65.

31. Karp JM, Leng Teo GS. Mesenchymal stem cell homing: the devil is in the details. Cell Stem Cell. 2009;4(3):206-16.

32. Lee RH, et al. Multipotent stromal cells from human marrow home to and promote repair of pancreatic islets and renal glomeruli in diabetic NOD/scid mice. Proc Natl Acad Sci U S A. 2006;103(46):17438-43.

33. Montanari S, et al. Mesenchymal stromal cells improve cardiac function and left ventricular remodeling in a heart transplantation model. J Heart Lung Transplant. 2015;34(11):1481-8,

34. Zhang $Y$, et al. Pterostilbene ameliorates nephropathy injury in Streptozotocin-induced diabetic rats. Pharmacology. 2019;104(1-2):71-80. 
35. Li Y, et al. Early intervention with mesenchymal stem cells prevents nephropathy in diabetic rats by ameliorating the inflammatory microenvironment. Int J Mol Med. 2018;41(5):2629-39.

36. Ina $\mathrm{K}$, et al. Transformation of interstitial fibroblasts and tubulointerstitial fibrosis in diabetic nephropathy. Med Electron Microsc. 2002;35(2):87-95

37. Li JH, et al. Smad7 inhibits fibrotic effect of TGF-Beta on renal tubular epithelial cells by blocking Smad2 activation. J Am Soc Nephrol. 2002;13(6): 1464-72.

38. Cahill EF, et al. Hepatocyte growth factor is required for mesenchymal stromal cell protection against bleomycin-induced pulmonary fibrosis. Stem Cells Transl Med. 2016;5(10):1307-18.

39. Bi B, et al. Stromal cells protect against acute tubular injury via an endocrine effect. J Am Soc Nephrol. 2007;18(9):2486-96.

40. Nimsanor $\mathrm{N}$, et al. Overexpression of anti-fibrotic factors ameliorates antifibrotic properties of Wharton's jelly derived mesenchymal stem cells under oxidative damage. Biosci Trends. 2019;13(5):411-22.

\section{Publisher's Note}

Springer Nature remains neutral with regard to jurisdictional claims in published maps and institutional affiliations.

Ready to submit your research? Choose BMC and benefit from:

- fast, convenient online submission

- thorough peer review by experienced researchers in your field

- rapid publication on acceptance

- support for research data, including large and complex data types

- gold Open Access which fosters wider collaboration and increased citations

- maximum visibility for your research: over $100 \mathrm{M}$ website views per year

At BMC, research is always in progress.

Learn more biomedcentral.com/submissions 